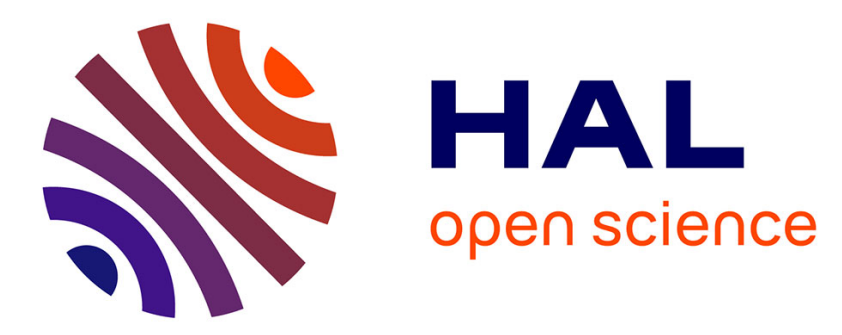

\title{
Automated detection of microaneurysms using robust blob descriptors
}

Kedir Adal, Sharib Ali, Désiré Sidibé, T.P. Karnowski, Edward Chaum, Fabrice Mériaudeau

\section{- To cite this version:}

Kedir Adal, Sharib Ali, Désiré Sidibé, T.P. Karnowski, Edward Chaum, et al.. Automated detection of microaneurysms using robust blob descriptors. SPIE Medical Imaging - Computer-Aided Diagnosis, Feb 2013, Orlando - FL, United States. pp.8670-22. hal-00784580

\section{HAL Id: hal-00784580 \\ https://u-bourgogne.hal.science/hal-00784580}

Submitted on 4 Feb 2013

HAL is a multi-disciplinary open access archive for the deposit and dissemination of scientific research documents, whether they are published or not. The documents may come from teaching and research institutions in France or abroad, or from public or private research centers.
L'archive ouverte pluridisciplinaire HAL, est destinée au dépôt et à la diffusion de documents scientifiques de niveau recherche, publiés ou non, émanant des établissements d'enseignement et de recherche français ou étrangers, des laboratoires publics ou privés. 


\title{
Automated Detection of Microaneurysms Using Robust Blob Descriptors
}

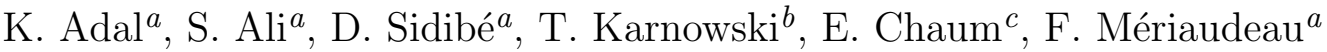 \\ ${ }^{a}$ Université de Bourgogne, France. \\ ${ }^{b}$ Oak Ridge National Laboratory, Oak Ridge TN, USA. \\ ${ }^{c}$ University of Tennessee Health Science Center, Memphis TN 38163 USA.
}

\begin{abstract}
Microaneurysms (MAs) are among the first signs of diabetic retinopathy (DR) that can be seen as round dark-red structures in digital color fundus photographs of retina. In recent years, automated computer-aided detection and diagnosis (CAD) of MAs has attracted many researchers due to its low-cost and versatile nature. In this paper, the MA detection problem is modeled as finding interest points from a given image and several interest point descriptors are introduced and integrated with machine learning techniques to detect MAs. The proposed approach starts by applying a novel fundus image contrast enhancement technique using Singular Value Decomposition (SVD) of fundus images. Then, Hessian-based candidate selection algorithm is applied to extract image regions which are more likely to be MAs. For each candidate region, robust low-level blob descriptors such as Speeded Up Robust Features (SURF) and Intensity Normalized Radon Transform are extracted to characterize candidate MA regions. The combined features are then classified using SVM which has been trained using ten manually annotated training images. The performance of the overall system is evaluated on Retinopathy Online Challenge (ROC) competition database. Preliminary results show the competitiveness of the proposed candidate selection techniques against state-of-the art methods as well as the promising future for the proposed descriptors to be used in the localization of MAs from fundus images.
\end{abstract}

Keywords: Microaneurysm, SURF, SVD, Hessian, SVM

\section{INTRODUCTION}

Diabetic Retinopathy (DR) which damages the retinal blood vessels is among the most common blindness causes in the developed country. The problem is further complicated by the fact that DR does not exhibit any distinctive symptoms which the patient can easily perceive until a severe stage is reached. Therefore, regular eye check-up and timely treatment is needed. However, the lack of specialized ophthalmologists together with associated higher medical costs makes regular check up costly. To fill this gap, development of low cost and versatile Computer Aided Diagnosis (CAD) systems, which can be used in clinical environments, have drawn much more attention in recent years. ${ }^{1}$

Microaneurysms (MAs) are among the early symptoms of DR, thus automated detection of MAs is among the different approaches used in public diabetic retinopathy screening systems. However, the variations in size, shape, presence of other retina vascular structures, and illumination variation of fundus images makes design of accurate CAD system very challenging.

In this paper, a specific automated DR diagnosis and detection system is developed for the detection of MAs. The proposed system is built based on well known low-level robust image descriptor extraction techniques so as to capture the subtle nature of MAs against the surrounding tissues. The different components of the overall system which is built using state of the art image processing and machine learning techniques are thoroughly discussed in the methodology section. 


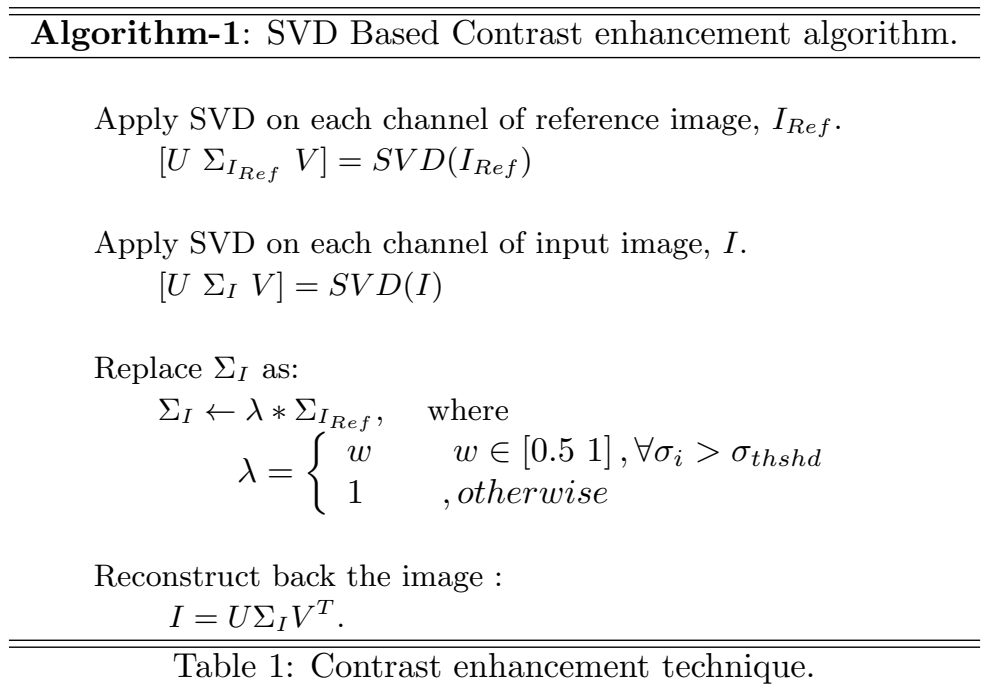

\section{METHODOLOGY}

\subsection{Pre-Processing}

Due to the external lighting source, color fundus images suffer from variation in illumination change, contrast, and shading. Contrast enhancement and shading removal is thus required before subsequent image analysis. The conventional approach is to apply either local or global histogram equalization techniques. However, these contrast enhancement techniques either does not give satisfactory result or they are prone to local noise enhancement.

In this paper, we proposed a new contrast enhancement technique which can reduce the shading effect while increasing the contrast of fundus images using Singular Value Decomposition (SVD). The main idea behind this approach is to analyze the intensity distribution of pixels indirectly from the singular values instead of using direct intensity histogram information. It is observed that the singular value distribution of good quality retinal images is equivalent but the corresponding distribution of low quality fundus images is low compared to the good ones. Figure 1 shows the green channel singular value distribution of two good quality images and one poor quality image. These distributions show that fundus images with good intensity contrast have somehow closer singular values whereas images with low contrast has smaller singular values compared to good quality images.

The proposed contrast enhancement method is done by replacing all the singular values of any low quality image by the corresponding values of the reference image. The replacement is done in a weighted manner to avoid any over-saturation effect which might be introduced when replacing very high singular values. The overall algorithm is summarized in table 1. Sample contrast enhancement result is also shown in figure 2.

\subsection{Candidate Selection}

Microaneurysm candidate extraction is the first crucial part in automated detection of diabetic retinopathy. Proper selection of possible suspicious regions reduces false detection rates as well as computation time; thus it speeds up the automated detection process.

Towards this end, we have developed a new candidate selection technique using scale-space approach. The proposed approach takes advantage of the Hessian operator on the GREEN channel of fundus images to extract circular-dark regions which have strong derivatives along both orthogonal directions. Such image regions can be selected by computing the eigenvalues of Hessian matrix at scale $\sigma=2$ for each pixel and selecting regions where $\lambda_{1}>0, \lambda_{2}>0$, and $\lambda_{1} \simeq \lambda_{2}$. Table 2 summarizes the proposed candidate selection algorithm. The main reason why we chose $\sigma=2$ is because of the fact that the response of majority of microaneurysms at this scale is good enough to separate them from other image regions. Moreover, at this stage we only need to roughly select 


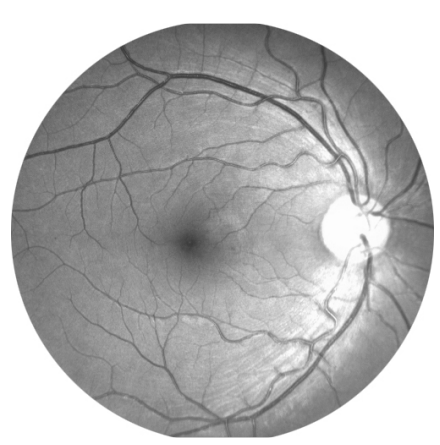

(a) Reference image-1.

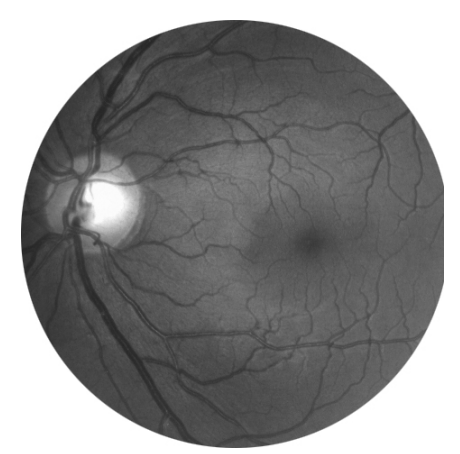

(b) Reference image-2.

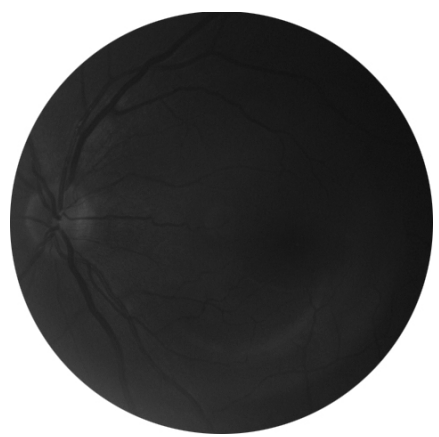

(c) Low contrast image.

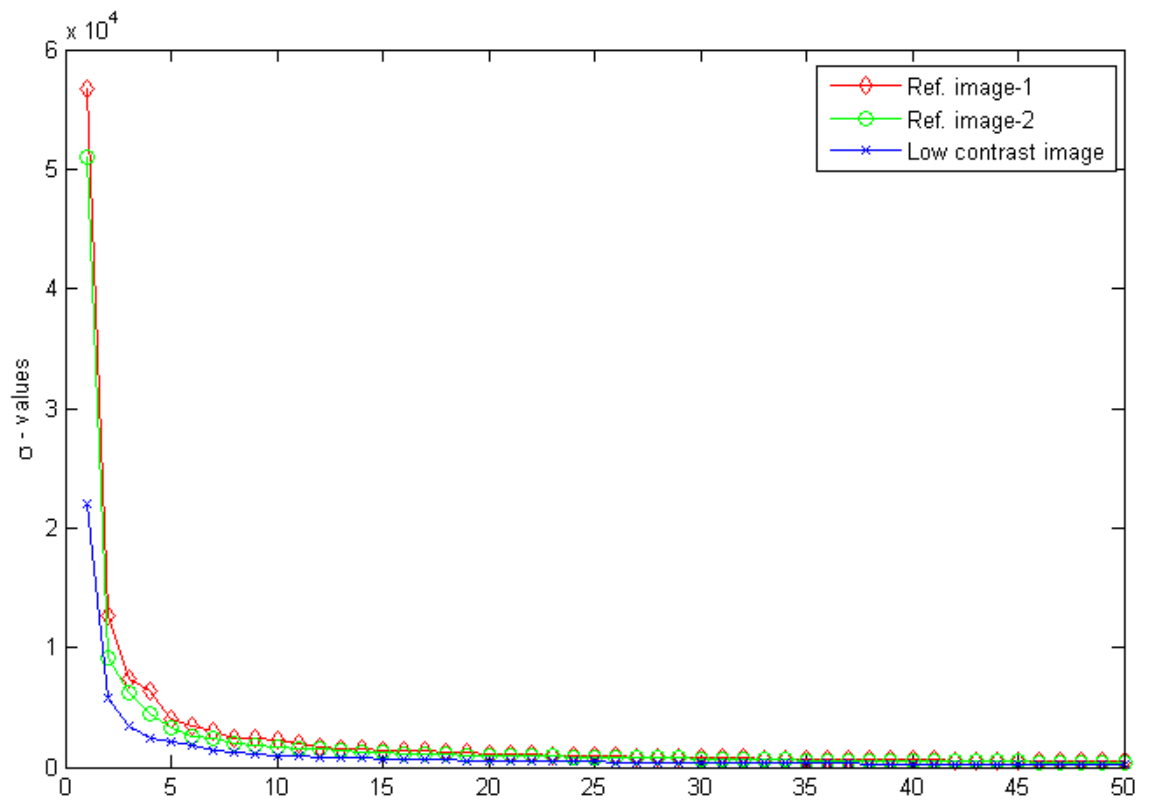

(d) Singular values of images (a)-(c).

Figure 1: Singular value distribution of different contrast fundus images.

out possible candidates for the next classification stage. The same scale has also been used by Niemeijer et al. ${ }^{2}$ to extract features of candidate microaneurysms.

The proposed candidate selection approach does not require vessel segmentation algorithm. This is mainly because blood vessels have strong derivative along one direction than the other, thus simple thresholding of the absolute ratio, $A r$, parameter allows to separate blood vessels from other regions. Figure 3 shows the result of the proposed candidate selection method.

\subsection{Feature Extraction}

For each selected candidate region within the fundus images, a robust well-known image descriptors called SURF are constructed. ${ }^{3}$ The two main operations in computing SURF are interest point detection and descriptor extraction. Since the interest points are detected using the aforementioned candidate selection algorith, we directly extract SURF descriptors for each candidate MA region. This is done by dividing each interest point region in $4 \times 4$ sub-windows and computing the Haar-wavelet response within each sub-window. These descriptors are $\Sigma d x, \Sigma d y, \Sigma|d x|$, and $\Sigma|d y|$. For each candidate MA, a total of 64 -SURF descriptors which characterize region of interest are extracted. A fixed scale of 1.6 is used to determine the size of the window to compute these descriptors. 


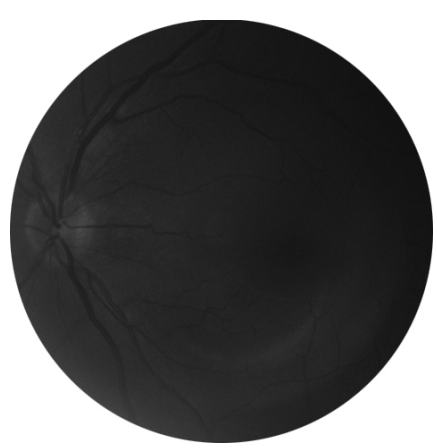

(a)

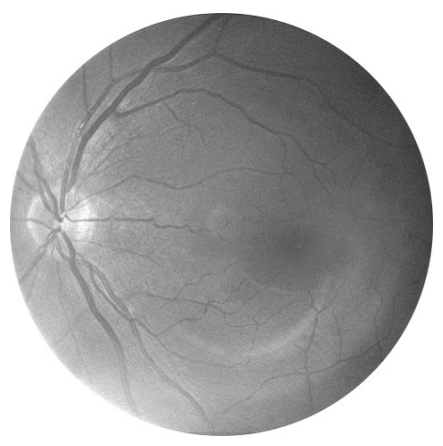

(b)

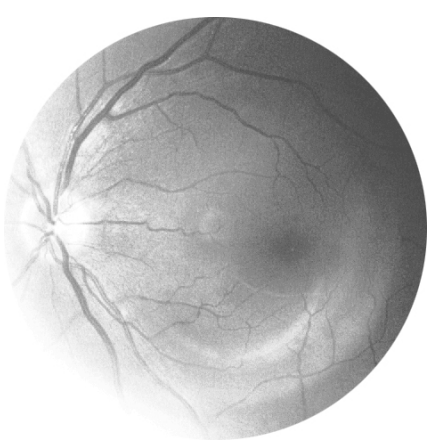

(c)

Figure 2: Example of contrast enhanced image: (a)Input image. (b)Proposed method. (c) Global histogram Equalization.

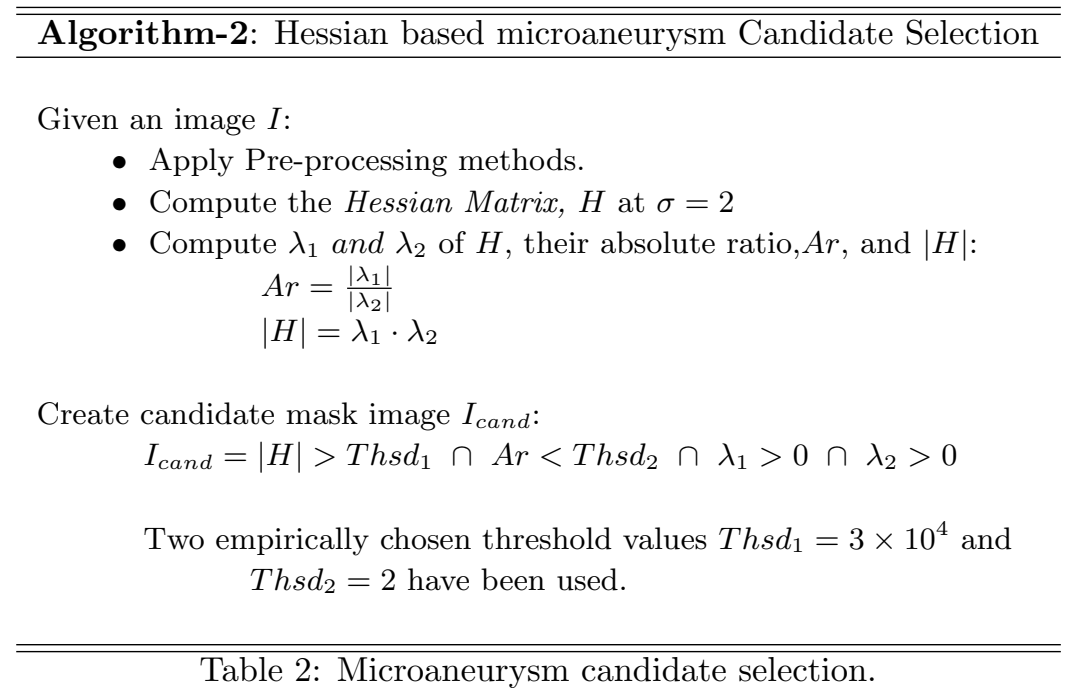

Previous works ${ }^{4}$ showed that Radon Transform can be used to detect MAs by applying directly on the original fundus images. We extend the same approach by applying Radon Transform on the Intensity Normalized image instead of the original image in order to make the Radon features illumination invariant. Intensity normalization is done by subtracting the mean intensity of an image patch from each pixel and dividing by standard deviation.

The Radon transform is then computed from the inverted green channel using a $15 \times 15$ image patch centered at each candidate MA and a projection angle of $\theta \in\left[\begin{array}{ll}0 & 180)\end{array}\right)$ with a resolution of $\theta_{\text {res }}=5^{\circ}$. Radon metrics that characterize circular-round structures are then computed as follows:

- Mean, $R_{\mu}(x)$, of each projection ray, $x$, at different projection angles:

$$
R_{\mu}(x)=\frac{1}{N} \Sigma_{n=1}^{N} R\left(n \cdot \theta_{\text {res }}, x\right), 1 \leq x \leq \rho,
$$

where $\rho$ is the number of projection rays and $N=\frac{180}{\theta_{\text {res }}}$ is the total number of projection angles. $R(\theta, x)$ refers to the Radon transform of ray $x$ at orientation angle $\theta$.

- Standard deviation, $R_{\sigma}(x)$ :

$$
R_{\sigma}(x)=\sqrt{\frac{1}{N} \Sigma_{n=1}^{N}\left[R\left(n \theta_{r e s}, x\right)-R_{\mu}(x)\right]^{2}} .
$$

The following 3 features are extracted from Radon space: 


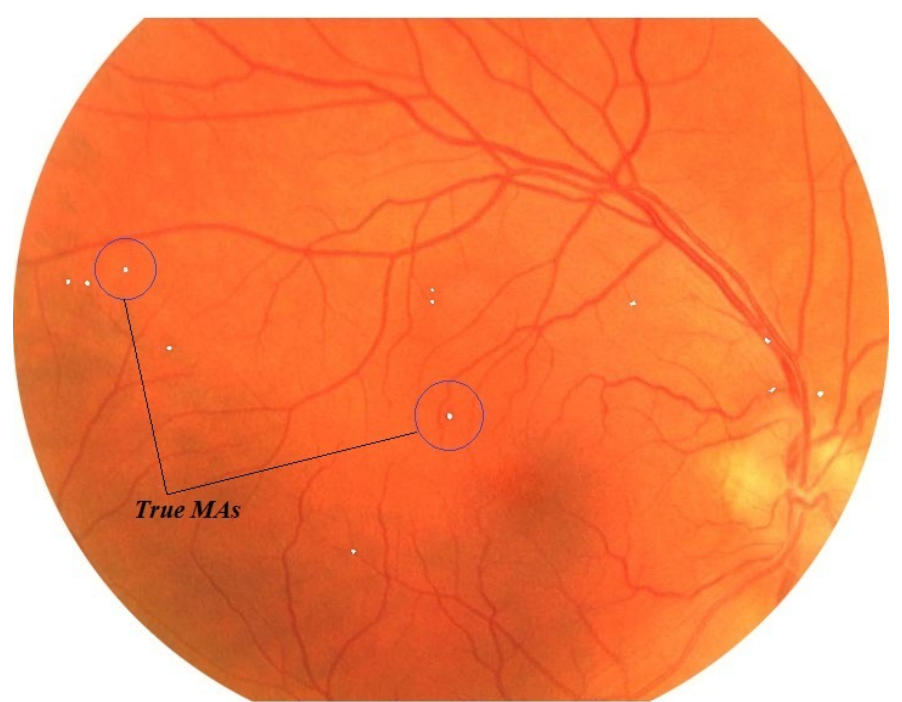

Figure 3: Selected MA candidates (White pixels) using proposed method.

- $\min \left\{R_{\mu}(x)\right\}$

- $\max \left\{R_{\sigma}(x)\right\}$

- $\sigma_{R_{\mu}(x)}$

In addition to the above Radon features, the following two additional features are computed directly from each image patch centered at location of interest:

- $\sigma_{I_{\text {patch }}}$ : the standard deviation of the image-patch (computed from the green channel).

- $n P$ :the number of pixels which are similar to the center pixel at the location of interest. This is found by applying region growing.

\subsection{Microaneurysm Classification}

In the final classification phase, SVM classifier is trained using ten manually annotated fundus images from our database. In training, POSITIVE samples are taken from the ground-truth annotations of training images whereas NEGATIVE samples are gathered by applying the proposed candidate selection method on those images and excluding all POSITIVE samples from all candidates. Finally Principal Component Analysis(PCA) is applied keeping $98 \%$ of the variance to train the classifier.

\section{RESULTS}

The overall performance of the proposed automatic MA detection is evaluated on the Retinopathy Online Challenge $(R O C)$ competition public database training and testing images. Using the training images as a test data-set, the candidate selection algorithm performance reaches a sensitivity of $44 \%$ at an average of 35 falsepositives (FPs) per image which is competitive against published methods ${ }^{5}$ as shown in table 3 . The proposed method reduces the false candidate by about 52 compared to the best performing technique proposed by Lazar et al. ${ }^{6}$ with a comparable sensitivity of $44.64 \%$.

Following our own evaluation on the training data-set, we have also participated in ROC-online challenge by submitting the performance results on the testing data-set. A competition performance measure (CPM) of 0.282 , which is computed by taking the average sensitivities at FPs ratio of $\left\{\frac{1}{8}, \frac{1}{4}, \frac{1}{2}, 1,2,4,8\right\}$, is achieved. The overall performance is shown in figure 4. In unoptimized Matlab implementation on a PC featuring a $2.00 \mathrm{GHz}$ Intel Core 2 Duo CPU, it takes an average of 15 seconds to analyze an image. 
Table 3: Comparison of different candidate extractors. Note that, except our method all the performance values are reported from Antal et al. ${ }^{5}$ implementation of each method.

\begin{tabular}{|c|c|c|c|}
\hline Authors & Methodology & Sensiti & $\overline{\mathrm{FPs} / \mathrm{I}}$ \\
\hline Spencer et al. ${ }^{7}$ & Top-hat transform & $12 \%$ & 20.3 \\
\hline Abdelazeem $^{8}$ & Circular Hough-transform & $28 \%$ & 505.85 \\
\hline Walter et al. $^{9}$ & Diameter closing & $36 \%$ & 154.42 \\
\hline Zhang et al. ${ }^{10}$ & Multiple-Gaussian mask & $33 \%$ & 328.3 \\
\hline Lazar et al. ${ }^{6}$ & Cross-section profile & $48 \%$ & 73.94 \\
\hline Our method & Hessian Operator & 44.64 & 35.20 \\
\hline
\end{tabular}

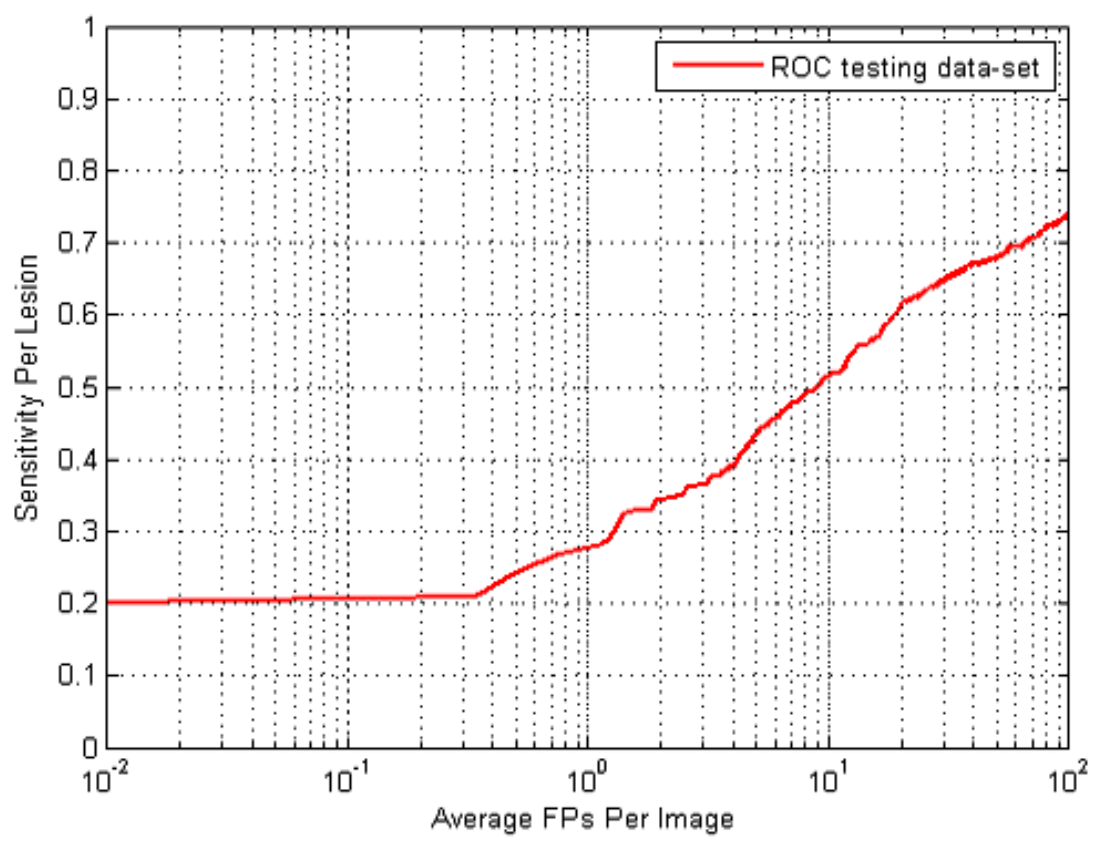

Figure 4: Performance results on ROC-testing data-set.

\section{CONCLUSION}

In this paper, an automated early diabetic retinopathy diagnosis system which can be used in public DR screening programs is presented. The system allows automated detection of MAs which are early symptoms of diabetic retinopathy. The proposed approach is built up on robust MA descriptors such as SURF and Radon Transform features followed by pattern recognition and classification technique. Moreover, a novel color fundus image contrast enhancement and candidate MA selection methods are proposed as a pre-processing step.

An overall performance score of 0.282 which has been achieved when evaluated on Retinopathy Online Challenge $(R O C)$ competition public database has shown the feasibility of the proposed low-level robust image descriptors to be used in the localization of MAs from fundus images. The proposed descriptors are especially advantageous in the analysis of fundus images where illumination variation and noise are prevalent challenges. Moreover, the fast computation time of the descriptors speeds-up the overall fundus image analysis.

To avoid the limitation of fixed scale usage in SURF descriptor extraction, an automatic local-scale estimation of the selected candidate MAs is currently being investigated. The possibility of combined the proposed approach together with other existing methods will also be our future work. 


\section{REFERENCES}

[1] Abràmoff, M., Garvin, M., and Sonka, M., "Retinal imaging and image analysis," Biomedical Engineering, IEEE Reviews in 3, 169-208 (2010).

[2] Niemeijer, M., Van Ginneken, B., Staal, J., Suttorp-Schulten, M., and Abràmoff, M., "Automatic detection of red lesions in digital color fundus photographs," Medical Imaging, IEEE Transactions on 24(5), 584-592 (2005).

[3] Bay, H., Ess, A., Tuytelaars, T., and Van Gool, L., "Speeded-up robust features (surf)," Computer Vision and Image Understanding 110(3), 346-359 (2008).

[4] Giancardoa, L., Meriaudeaub, F., Karnowskia, T., Tobina, K., Lic, Y., and Chaum, E., "Microaneurysms detection with the radon cliff operator in retinal fundus images.," in [Proc. of SPIE Vol], 7623, 76230U-1 (2010).

[5] Antal, B. and Hajdu, A., "An ensemble-based system for microaneurysm detection and diabetic retinopathy grading.," IEEE Transactions on Bio-medical Engineering (2012).

[6] Lazar, I., Hajdu, A., and Quareshi, R., "Retinal microaneurysm detection based on intensity profile analysis," in [8th International Conference on Applied Informatics], (2010).

[7] Spencer, T., Phillips, R., Sharp, P., and Forrester, J., "Automated detection and quantification of microaneurysms in fluorescein angiograms," Graefe's archive for clinical and experimental ophthalmology 230(1), 36-41 (1992).

[8] Abdelazeem, S., "Micro-aneurysm detection using vessels removal and circular hough transform," in [Radio Science Conference, 2002. (NRSC 2002). Proceedings of the Nineteenth National], 421 - 426 (2002).

[9] Walter, T., Massin, P., Erginay, A., Ordonez, R., Jeulin, C., and Klein, J.-C., "Automatic detection of microaneurysms in color fundus images," Medical Image Analysis 11(6), 555 - 566 (2007).

[10] Zhang, B., Wu, X., You, J., Li, Q., and Karray, F., "Detection of microaneurysms using multi-scale correlation coefficients," Pattern Recognition 43(6), 2237-2248 (2010). 